\title{
WG2 Summary: Low-x and Diffraction
}

\section{Néstor Armesto*}

Instituto Galego de Física de Altas Enerxías IGFAE, Universidade de Santiago de Compostela, 15782 Santiago de Compostela, Galicia-Spain

E-mail: nestor.armesto@usc.es

\section{Robert Ciesielski}

The Rockefeller University, 1230 York Avenue, New York, NY 10065, USA

E-mail: robert.ciesielski@rockefeller.edu

\section{Paul Richard Newman}

School of Physics and Astronomy, University of Birmingham, Edgbaston Park Rd., Birmingham B15 2TT, UK

E-mail: paul.richard.newman@cern.ch

Here we summarise the contributions to Working Group 2: Low- $x$ and Diffraction, at the DIS2019

Workshop.

XXVII International Workshop on Deep-Inelastic Scattering and Related Subjects - DIS2019

8-12 April, 2019

Torino, Italy

*Supported by Ministerio de Ciencia e Innovación of Spain under projects FPA2017-83814-P and Unidad de Excelencia María de Maetzu under project MDM-2016-0692, by Xunta de Galicia under project ED431C 2017/07, and by FEDER. This work has been performed in the framework of COST Action CA15213 "Theory of hot matter and relativistic heavy-ion collisions" (THOR). 


\section{Introduction}

At DIS2019, many new ideas and experimental results were presented and discussed. 37 talks (24 theoretical and 13 experimental ones) were given in the sessions corresponding to Working Group 2: Low- $x$ and Diffraction. Five theoretical talks were presented in the joint session WG2 + WG7: Future of DIS, with four of them covered in this summary. And four talks (two theoretical and two experimental) were given in the joint session WG2 + WG4: Hadronic and Electroweak Observables, with the two theoretical talks covered here. We start by summarising the theory contributions in Section 2, to continue with the experimental ones in Section 3. All talks can be found in [1]. We refer to the corresponding contributions when they are available at the time of writing this summary, otherwise mentioning the relevant papers.

\section{Theory talks}

An impressive progress in several theoretical aspects in the field was shown in this conference, with contributions ranging from the treatment of the formalism to comparisons with experimental data. In order to organise the presentations, theory talks have been loosely classified subject-like into those related with inclusive cross sections, those dealing with evolution equations, those facing problems in particle production, and more formal theory talks.

\subsection{Inclusive cross sections}

The talks by G. Beuf and R. Paatelainen were devoted to the calculation of the DIS cross section with massive quarks (required to compare with data on charm and beauty DIS cross sections) at next-to-leading order (NLO) in Light Cone Perturbation Theory (LCPT), suitable for the inclusion of BFKL and non-linear dynamics. The first talk dealt with a long standing problem of mass renormalization at NLO on the light front, with two different quark mass corrections appearing in vertex and propagator (the second one different from the result in covariant perturbation theory) and a gluon mass correction. It was shown how this problem is solved and the results become compatible with covariant perturbation theory, with no gluon mass counterterm required. Using this theoretical development, the second talk presented the results for the longitudinally polarised DIS cross section.

A. Watanabe [2] presented a model for hadronic cross sections based on holographic ideas. The model contains gravitational form factors fixed by hadronic spectra, and a Reggeon propagator with three parameters to be fitted. It was shown to give a reasonable description of the total and differential elastic cross sections at LHC energies, including recent TOTEM data.

Finally, C. Ewerz presented a model for DIS cross sections with hard and soft tensor Pomerons, and an $f_{2}$ Reggeon [3]. With 25 parameters the model provides a good description of small- $x$ HERA data down to photoproduction, with a sizeable soft contribution up to $Q^{2}=20 \mathrm{GeV}^{2}$.

\subsection{Evolution equations}

Two talks dealt with the known problems found in the NLO Balitsky-Kovchegov (BK) equation. D. Triantafyllopoulos recalled how the existence of large $\ln ^{2}$ terms motivated resummation. When the evolution is performed from projectile to target and then corrected for lifetime ordering, 
the results are known to be unstable and require fine tuning. In the talk it was shown that performing the evolution from target to projectile and correcting for ordering in the plus light-cone momenta leads to more stable results [4]. This is a step towards finding the right evolution variable unifying DGLAP and small- $x$ non-linear dynamics. Besides, M. Matas showed that the Coulomb tails at large impact parameters generated by BK evolution which lacks confinement dynamics, are killed when the mentioned resummation effects are introduced. This fact was then employed to provide a description of small- $x$ HERA data in the dipole model [5].

B. Ermolaev showed the results for the $F_{1}$ structure function in the double leading logarithmic approximation (DLA) which resums terms $\propto \alpha_{s} \ln ^{2} x$. These terms are usually neglecting compared to those $\propto \alpha_{s} \ln x$ (leading logarithmic or BFKL approximation). This approximation, previously employed for function $g_{1}$, is shown to lead to scaling in $Q^{2} / x^{2}$ and a Pomeron-like behaviour with intercept smaller than that in BFKL, and to compete with BFKL down to small values of $x$ [6].

Finally, G. Chirilli discussed the non-eikonal corrections, fundamental for spin physics, in the high-energy formalism by Balitsky. He presented [7] evolution equations for flavour nonsinglet and flavour singlet polarised structure functions that coincide with those obtained by Bartels, Ermolaev and Ryskin in DLA.

\subsection{Particle production}

Concerning particle production, there were several talks related with the possibilities of unraveling new QCD dynamics through the study of correlations. First, A. Papa discussed the NLO BFKL effects on heavy quark azimuthal correlations at large rapidity separations, in photon-photon collisions at very large energies, $\mathscr{O}(1 \mathrm{TeV})$. NLO effects turn out to be very sizeable, and the extension to hadroproduction was discussed [8]. F. Celiberto [9] discussed the NLO effects on the azimuthal decorrelation of rapidity separated hadrons and jets, in the forward direction in pp collisions at LHC energies. NLO BFKL results showed a large decorrelation compared to DGLAP results. K. Golec-Biernat proposed the use of the helicity dependence in DY pair-jet correlations that show a larger decorrelation than jet-jet (Mueller-Navelet) correlations [10]. Finally, A. Ramnath showed results on the rapidity evolution of a rapidity separated quark-gluon system, described by JIMWLK evolution in the dense regime that reduces to BFKL when the system becomes dilute [11]. This is a first step toward the calculation of density non-linear effects on Mueller-Navelet jets and other observables sensitive to small- $x$ dynamics.

Turning to more fundamental aspects of particle production, K. Tu discussed how the description of hadrons in the parton model as an incoherent superposition of partons can be reconciled with the fact that the hadron is a pure quantum state. He showed the attempts to check an earlier proposal that the entanglement entropy should be proportional to the logarithm of the gluon momentum density in DIS and hadronic collisions. In DIS, a comparison with Monte Carlo results failed while the trend in pp collisions at the LHC is in agreement with the expectation [12].

Coming back to particle correlations, specifically to the azimuthal correlations observed in $\mathrm{pp}$ and $\mathrm{pA}$ at the LHC - the ridge, D. Wertepny discussed a possible initial state origin of azimuthal correlations coming from the fact that hadrons may have a partonic structure more involved than commonly assumed. In the framework of the McLerran-Venugopalan model used in those initial state calculations, he showed how the hadron colour charge distribution gets a tensorial, domainlike anisotropic structure when the colour neutralisation scale is of the order or larger than the 
hadron size. Such anisotropic structures have been used without further justification to explain the observed azimuthal structures. Additionally, usual models for the hadron colour charge distribution contain intrinsically a symmetry between the near and away side regions and, therefore, cannot generate odd azimuthal harmonics. T. Altinoluk showed how this symmetry is linked to the use of the eikonal approximation and how non-eikonal corrections (those stemming from the relaxation of the treatment of the colliding hadrons as shockwaves) affect particle production and correlations in a way that such odd harmonics are generated but become washed out with increasing energy. He proposed the used of a modified expression for the Lipatov vertex in order to take into account these non-eikonal corrections [13].

Two talks were devoted to ongoing developments of the formalism. Y. Mulian showed steps towards the calculation of the full NLO forward dijet production in pA collisions [14]. Several experiments at the LHC are or will be measuring such cross section. Therefore, this extremely involved calculation in LCPT deals with a most important observable for establishing the relevance of saturation at the LHC and the link with TMD factorisation. On the other hand, R. Maciula showed the progress in the calculation of quarkonium production in hadronic collisions within $k_{T^{-}}$ factorisation for heavy quark generation and the Color Evaporation Model to produce the bound states [15]. He showed that while sizeable scale, mass and parton density uncertainties exist, a good description of data in pp collisions at the LHC can be achieved.

Two more talks revised the situation with double parton scattering DPS. First, the general review [16] by J. Gaunt illustrated several important recent progresses in the field. The definition of the DPS cross section has been revised and a regulation to avoid double counting with single parton scattering has been proposed. Several observables that are very sensitive to DPS were revised, together with the extension of the formalism to account for transverse momenta. The delicate interplay of spin, colour and flavour was discussed, and the link to lattice calculations, the implementation in Monte Carlo showers and the different effects in pA scattering. On the other hand, J.-P. Lansberg discussed the status of the extraction of the effective DPS cross section from experiment. He pointed out that those extractions based of quarkonium observables give systematically lower values than all other extractions, and suggested possible explanations related with flavour dependence or with the fact that such observables are measured at non-central rapidities, contrary to non-quarkonium observables. To conclude with DPS, L. Motyka analysed the effect of DPS in the form of two BFKL pomeron exchange, on quarkonium production in the Color Singlet Model. The effects on the production cross section in hadronic collisions, including pomeron loops, seems small [17].

Turning now to diffraction, a review was provided by M. Strikman. He pointed out the success of the standard collinear framework to describe parton densities in nuclei when supplemented with information from diffraction in $e$ p from HERA - leading twist nuclear shadowing. The obtained nuclear parton densities provide a good description of quarkonium production as measured in Ultra Peripheral Collisions (UPCs) on nuclear targets at the LHC. They seem not to require a fast approach to the black disk limit that is usually associated with saturation. He also underlined the importance of cross section (colour) fluctuations on top of geometrical fluctuations as addressed by the usual Glauber picture, to describe elastic quarkonium production on nuclear targets as measured in UPCs at RHIC and the LHC. He proposed the study of rapidity gap events with fixed diffractive mass to test the high-energy dynamics of QCD. He provided definite predictions on the behaviour 
of exclusive diffractive cross section on nuclear targets with energy and exchanged momentum.

From analysing the BK equation for diffraction, S. Munier established predictions and an equation for the distribution of the rapidity gap in diffractive events. He also discussed its analogy with the time distribution of splittings in random walks, a most interesting link between highenergy and statistical physics [18]. On the other hand, C. Flett [19] discussed the current efforts to reduce the theoretical uncertainties, mainly due to the scale choice, in NLO calculations of exclusive vector meson production in UPCs. Such studies are required before such observable can be used to reliably extract parton densities.

Finally, there were two talks on diffractive dijets production in photoproduction and DIS, an observable sensitive to linearly polarised gluons in the proton or nucleus. V. Skokov showed a Monte Carlo simulator for such observable, including small- $x$ evolution (JIMWLK) and hadronisation. He showed how acceptance must be carefully taking into account in order to reliably subtract the background for the azimuthal asymmetries that are proportional to the linearly polarised gluon density [20]. H. Mantyssari [21] showed a study of charm dijet azimuthal harmonics, including the effect of small- $x$ JIMWLK evolution. He discussed the relation with the Wigner distribution of partons in the hadron, a central quantity in hadronic physics with a direct link to GPDs and TMDs, and how it could be extracted from experiment.

\subsection{Formalism}

There were two formal talks on recent progress dealing with Lipatov's effective action which aims to be the complete effective theory for high-energy QCD. S. Bondarenko formulated the effective action as a Reggeon Field Theory (RFT), which allows a connection with other formalisms like the high energy operator product expansion by Balitsky or the Color Glass Condensate. He discussed how the vertices of the effective action get both QCD and RFT corrections, how both corrections compete and showed the results for the one loop corrections to the reggeized gluon propagator [22]. M. Nefedov [23] showed the calculation of the one loop effective vertices in the RFT coming from Lipatov's effective action. He performed the involved exercise of cross-checking them with the standard QCD results in $\gamma^{*} \gamma$ scattering using the effective $\gamma^{*}$-reggeon-quark vertex.

\section{Experimental talks}

In this section we summarize the experimental resuts presented in the WG2 session. For ease of presentation, we group them into the following broad categories.

\subsection{Diffractive PDFs}

Two talks covered the subject of extraction of diffractive parton distribution functions (DPDFs) from the diffractive deep-inelastic scattering (DDIS) data.

R. Zlebcik presented [24] the new H1 fits of DPDFs to the HERA inclusive and dijet data in DDIS. Given a progress in theory calculations, where the next-to-next-to-leading order (NNLO) predictions are now available for both processes [25], the new DPDFs have been determined at NNLO level for the first time. Compared to previous H1 NLO fits [26], the current analysis includes the high-precision HERA-II data of the H1 collaboration, which corresponds to a 40-fold increase in luminosity for inclusive data and six-fold increase for dijet data. The latter help significantly 

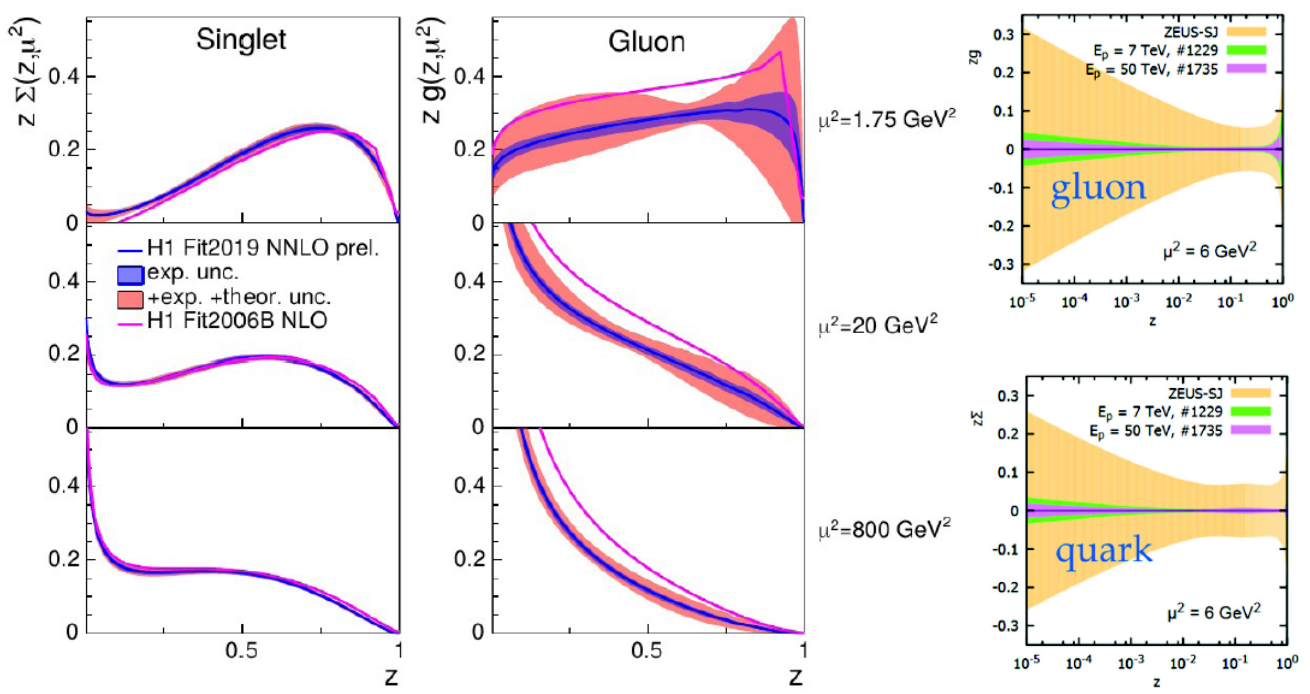

Figure 1: Left panel: quark $(\Sigma)$ and gluon $(g)$ distribution in the H1 NNLO Fit2019 DPDFs (blue line), shown with experimental and theory uncertainties, compared to the H1 NLO Fit2006B DPDFs (pink line). Right: Relative uncertainties of gluon (top) and quark (bottom) DPDFs from the diffractive QCD analysis of the LHeC (green) and FCC-eh (magenda) pseudodata, compared to those from the ZEUS-SJ DPDFs.

to constrain the gluon DPDFs. Fig. 1 (left) shows the extracted H1 NNLO DPDFs, compared to the previous NLO DPDF fits. While the sea quark DPDFs look quite similar at both NLO and NNLO, the gluon PDFs at NNLO are about $25 \%$ below those extracted at NLO. This drop is compensated by a higher gluon-induced cross section at the matrix-element level at NNLO [25]. The new NNLO DPDFs are used to predict total and differential dijet cross sections in DDIS at NNLO accuracy. These agree nicely with other HERA dijet measurements that were not included in the fit, suggesting that factorization in DDIS holds up to NNLO.

A. Stasto showed [27] a feasibility study of measurements of the proton DPDFs at possible future electron-proton machines at CERN, namely the Large Hadron-electron Collider (LHeC) and the Future Circular Collider (FCC-eh) [28]. These machines will allow to study DDIS in previously unexplored kinematic regions; the $\mathrm{LHeC}(60 \mathrm{GeV}$ electrons on $7 \mathrm{TeV}$ protons) will access the values of Bjorken $\mathrm{x}\left(Q^{2}\right)$ about 20 (200) times smaller (higher) than those reached at HERA; the FCC-eh $(60 \mathrm{GeV}$ electrons on $50 \mathrm{TeV}$ protons) will further enlarge this range by one additional order of magnitude in both $x$ and $Q^{2}$. Pseudodata in both the LHeC and FCC-eh kinematic ranges were generated based on NLO calculations of DDIS using extrapolated ZEUS-SJ DPDFs [29]. A uniform 5\% systematic uncertainty and a statistical uncertainty corresponding to an integrated luminosity of $2 \mathrm{fb}^{-1}$ were assumed. The pseudodata were then used to extract the DPDFs, which relative accuracy is shown in Fig. 1 (right). Compared to HERA analyses, the DPDFs precision is roughly increased by a factor of ten for the $\mathrm{LHeC}$ and by a factor of 20 for the FCC-eh. Moreover, a non-negligible diffractive contribution from the top quark is expected at the FCC-eh, allowing the first observation of the diffractively produced top quark. A similar study of the DPDFs in the electron-nucleus collisions at the $\mathrm{LHeC}$ and FCC-eh is ongoing.

\subsection{Total and elastic proton-proton cross section and odderon}

F. Nemes presented [30] a compilation of recent results of the TOTEM collaboration on elastic 

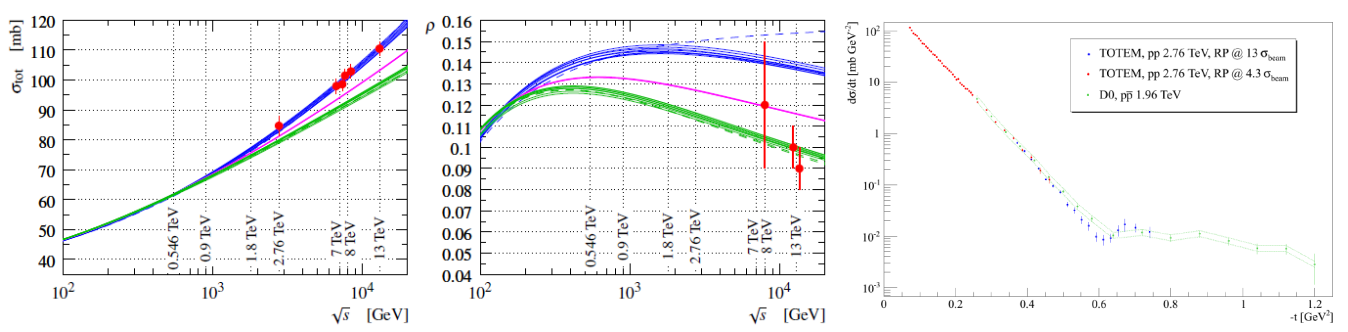

Figure 2: TOTEM measurements of the total hadronic pp cross section (left) and the $\rho$ parameter (middle) as a function of the pp center-of-mass energy, compared to various predictions of COMPETE collaboration. Right: the $d \sigma / d|t|$ cross section measured by TOTEM in $p p$ collisions at $2.76 \mathrm{TeV}$ and by D0 in $p \bar{p}$ collisions at $1.96 \mathrm{TeV}$ (green).

$(p p \rightarrow p p$ ) and total hadronic pp cross sections at the LHC. TOTEM measures elastic cross section by tagging protons in Roman Pot detectors (RPs), located at $z= \pm 220 \mathrm{~m}$ dowstream the CMS detector. The total pp cross section is obtained from elastic scattering using the optical theorem. The recent results include measurements at the $p p$ center-of-mass energy of $13 \mathrm{TeV}$, at lower [31], moderate [32], and higher [33] values of the 4-momentum transfer squared between the protons, $|t|$, and at $2.76 \mathrm{TeV}$ [34]. Fig. 2 (left) and (middle) show the total pp cross section and the ratio $\rho$ of the real to imaginary part of the hadronic forward $(t=0)$ amplitude as measured by TOTEM at different energies. The data are compared to various predictions of the COMPETE model [36] that describe the data at lower energies. A striking feature emerging from the comparison is that none of the predictions is able to simultaneously describe both quantities at the LHC energies. TOTEM interprets this as an indication of an additional contribution to the pp scattering at higher energies that comes from the exchange of the odderon - a three-gluon, colorless, odd charge-parity counterpart of the pomeron ( $C=+1$ two-gluon exchange). The existence of the odderon is further tested by comparing the $d \sigma / d|t|$ cross section measured by TOTEM in $p p$ collisions at $2.76 \mathrm{TeV}$ with a similar measurement by $\mathrm{D} 0$ in $p \bar{p}$ collisions at $1.96 \mathrm{TeV}$ [35] (Fig. 2 right). In models with pomeron exchange, an invariance of the $d \sigma / d|t|$ spectrum is expected for the $p p$ and $p \bar{p}$ scattering at the same energy, while the data (at similar energies) suggest a different behavior, in particular in the region around the diffractive peak. The significance of the odderon observation is currently being evaluated in a joint paper by TOTEM and D0.

An independent search for the odderon was proposed by A. Szczurek [37] in a talk on low-mass central exclusive production of $p \bar{p}$ and $\phi \phi$ pairs and single $\phi$ mesons in proton-proton collisions $(p p \rightarrow p p p \bar{p}, p p \rightarrow p p \phi \phi$ and $p p \rightarrow p p \phi)$. Calculations based on a tensor-pomeron model [38, 39] suggest that the odderon contribution may be clearly visible in the $\phi \phi$ channel, for high masses of the $\phi \phi$ pair (above $6 \mathrm{GeV}$ ) or a high rapidity separation between the two $\phi$ mesons.

\subsection{Diffraction in proton-proton collisions}

M. Tasevsky presented [40] a new ATLAS measurement of inclusive single-diffractive (SD) dissociation $(p p \rightarrow p X)$ with the scattered proton tagged in the ALFA spectrometer [41], located at $z= \pm 240 \mathrm{~m}$ dowstream the ATLAS detector. The measurement is based on the low-pileup 8 $\mathrm{TeV}$ data, taken in a special run with the $\beta^{*}=90 \mathrm{~m}$ beam optics. The inclusive SD cross section is measured as a function of the 4-momentum transfer squared, $|t|$, the fractional longitudinal momentum loss of the incoming proton, $\xi$, and the rapidity separation $\Delta \eta$ between the proton and the 

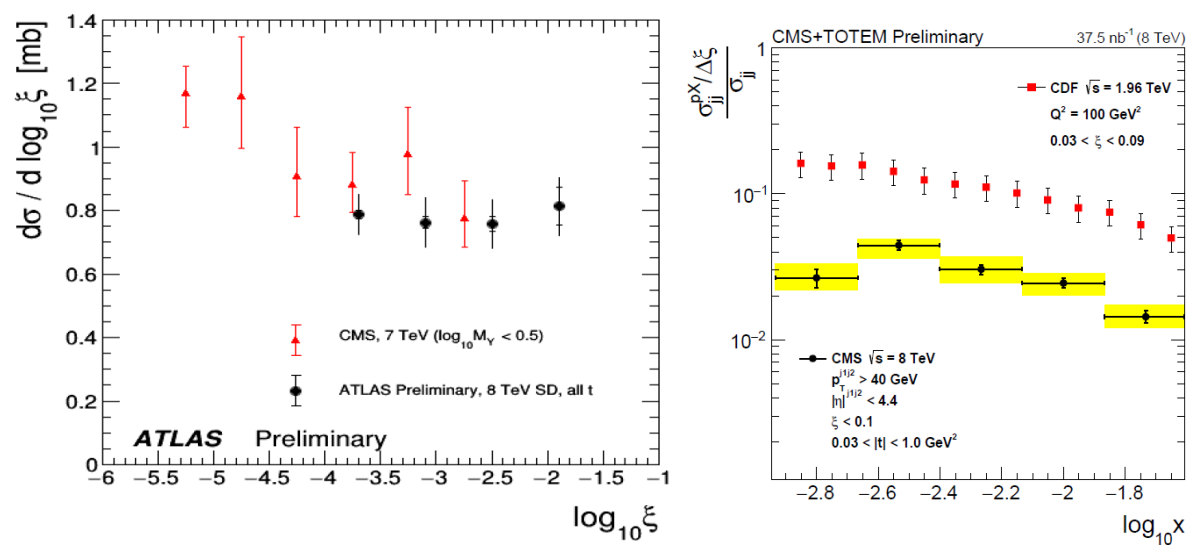

Figure 3: Left: Inclusive SD cross section at $8 \mathrm{TeV}$ as a function of $\log _{10} \xi$ measured by ATLAS using proton tag, compared to similar CMS result at $7 \mathrm{TeV}$ based on rapidity gap selection. Right: Ratio of SD to inclusive dijet cross sections measured in a joint CMS-TOTEM analysis at $8 \mathrm{TeV}$ using proton tag, compared to similar result by $\mathrm{CDF}$ at $1.96 \mathrm{TeV}$.

system $X$, for $0.016<-t<0.43 \mathrm{GeV}^{2}$ and $-4<\log _{10} \xi<-1.6$. A fit to the $d \sigma / d|t|$ with the exponential function $e^{b t}$ yields the slope $b=7.60 \pm 0.23$ (stat) \pm 0.22 (syst) $\mathrm{GeV}^{-2}$, in agreement with extrapolations from low-energy measurements. Fig. 3 (left) shows the measured SD cross section as a function of $\log _{10} \xi$, compared to the measurement by CMS at $7 \mathrm{TeV}$ based on a rapidity gap selection [42]. A good agreement between both results is found, after a small admixture of double-diffractive dissociation events $(p p \rightarrow X Y$ ) is subtracted from the CMS data.

Results on hard diffraction at the LHC were discussed in a presentation by K. Kuznetsova [43]. The talk covered a new measurement of the SD dijet cross section $(p p \rightarrow p j j X)$ with proton tag performed jointly by the CMS and TOTEM collaborations [44], and the CMS measurement of the production of jet-gap-jet events at $7 \mathrm{TeV}$ [46], sensitive to the BFKL dynamics. The SD dijet analysis is based on a dedicated low-pileup data at $8 \mathrm{TeV}$, with the dijets measured in the CMS detector and the scattered proton recorded in the TOTEM RPs. The SD dijet cross section is measured as a function of $|t|, \xi$ and $\beta=x / \xi$, for $0.03<-t<0.1 \mathrm{GeV}^{2}, \xi<0.1$ and jets with $p_{T}>40 \mathrm{GeV}$ and $|\eta|<4.4$. A fit with the exponential function $e^{b t}$ to the $d \sigma / d|t|$ cross section yields the slope $b=6.6 \pm 0.6$ (stat) \pm 0.09 (syst) $\mathrm{GeV}^{-2}$, in agreement with a similar CDF result at $1.96 \mathrm{TeV}$. Fig. 3 (right) shows the measured ratio of SD to inclusive dijet cross sections as a function of Bjorken- $x$, compared to the CDF data. A factor-of-three suppression of the ratio with collision energy can be observed, attributed to an enhanced contribution at $8 \mathrm{TeV}$ from soft rescattering processes that spoil the diffractive gap. A comparison of the measured SD dijet cross section to POMWIG predictions provides an estimate of the gap survival probability of $9 \pm 2 \%$. Fig. 4 (left) shows the ratio of jet-gap-jet to dijet events measured by CMS at $7 \mathrm{TeV}$, for jets with $p_{T}>40 \mathrm{GeV}$ and $|\eta|>1.5$, reconstructed in the opposite hemispheres of the detector. The data, shown as a function of jet $p_{T}$, are compared to Tevatron results from similar analyses at 0.63 and $1.8 \mathrm{TeV}$. A factor-of-two suppresion of the ratio at $7 \mathrm{TeV}$ relative to that at $1.8 \mathrm{TeV}$ can be seen. The CMS data are also compared (not shown) to the predictions of the NLL BFKL model by Ekstedt, Enberg and Ingelman [46], with different implementations of the contribution from soft rescattering processes.

Finally, C. Dilks showed [47] a new STAR result on the semi-inclusive $p^{\uparrow \downarrow} p \rightarrow p \pi^{0} X$ production in the SD process at $200 \mathrm{GeV}$, with the proton from the transversely polarized beam detected 

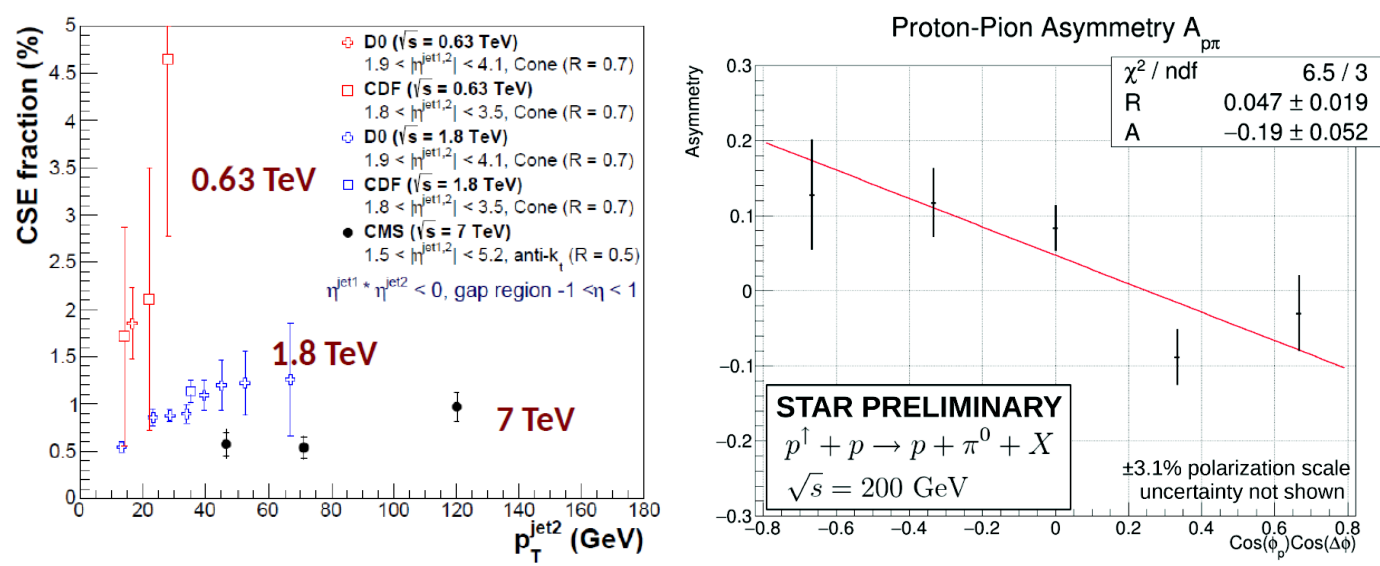

Figure 4: Left: Ratio of jet-gap-jet to dijet events (CSE fraction) as a function of jet $p_{T}$ measured by CMS at $7 \mathrm{TeV}$, compared to the Tevatron data at 0.63 and $1.8 \mathrm{TeV}$. Right: Transverse single-spin asymmetry measured by STAR at $200 \mathrm{GeV}$ for the $p^{\uparrow \downarrow} p \rightarrow p \pi^{0} X$ process.

in the STAR RPs and the $\pi^{0} \rightarrow \gamma \gamma$ measured in the direction of the scattered proton in the Forward Meson Spectrometer. The analysis shows that in about one percent of SD events the scattered proton is accompanied by the $\pi^{0}$, and that their energies add up to the incoming beam energy. For those events, single-spin asymmetries are studied as functions of various combinations of the azimuthal angle of the scattered proton, $\phi_{p}$, and the angle between the $\pi^{0}$ and the proton scattering place, $\Delta \phi$. A significant $-19 \% \pm 5.2 \%$ asymmetry is observed via a modulation in $\cos \phi_{p} \cos \Delta \phi$ (Fig. 4 right) when the $\pi^{0}$ is produced near the proton scattering plane. This production mechanism remains open to theory interpretation.

\subsection{Exclusive processes}

Nine talks covered several new results on exclusive vector-meson production in photon-proton and photon-nucleus collisions, and diphoton and dilepton production in photon-photon fusion.

A. Bolz presented [48] a new H1 measurement of exclusive and proton-dissociative photoproduction $\left(Q^{2}=0\right)$ of the $\rho^{0}$ meson using the high-statistics HERA-II data [49]. The separation of exclusive and proton-dissociative events was performed based on the presence or the absence of a signal in the Forward Tagging Station, sensitive to dissociated masses $M_{Y}$ up to $10 \mathrm{GeV}$. Cross sections measured in bins of the $\gamma p$ center-of-mass energy $W$ are shown in Fig. 5 (left). The data spans
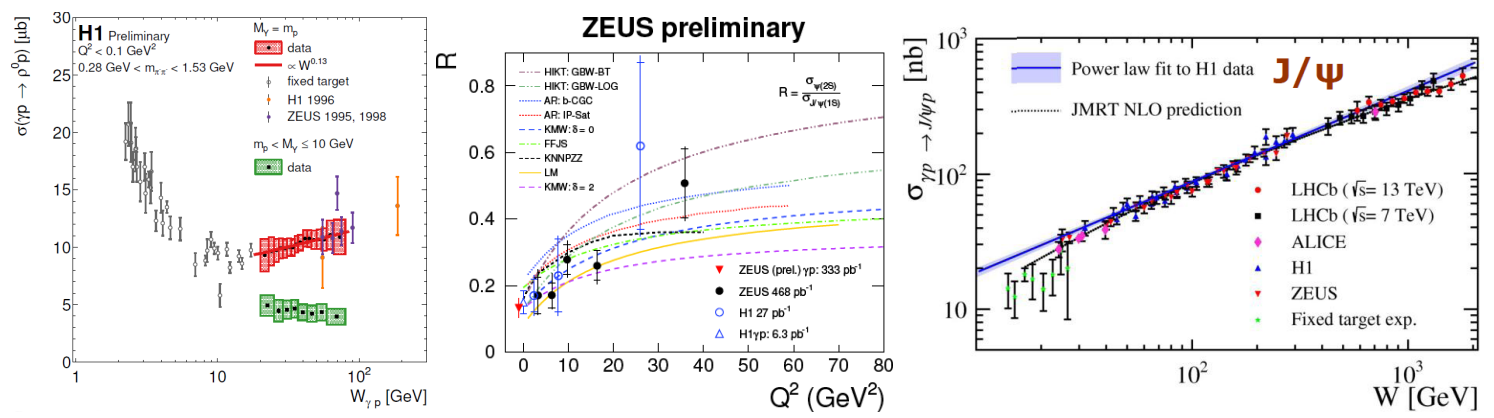

Figure 5: Left: Cross section of exclusive (red) and proton-dissociative (green, $\left.M_{Y}<10 \mathrm{GeV}\right) \rho^{0}$ production measured as a function of $\mathrm{W}$ by $\mathrm{H} 1$. Middle: the $\psi(2 S)$ to $J / \psi$ cross section ratio measured in bins of $Q^{2}$ by ZEUS. Right: exclusive $\gamma p \rightarrow J / \psi p$ cross section as a function of W measured by LHCb. 

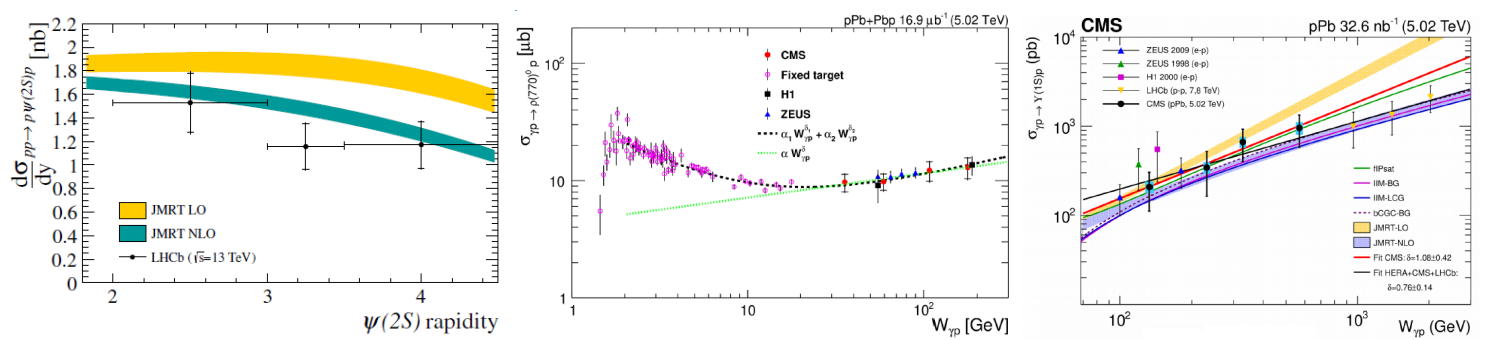

Figure 6: Left: exclusive $\psi(2 S)$ cross section in bins of rapidity measured by LHCb in pp collisions at 13 $\mathrm{TeV}$. Exclusive $\rho^{0}$ (middle) and $\Upsilon$ (right) cross section vs $\mathrm{W}$ measured in $\mathrm{pPb}$ collision at $5 \mathrm{TeV}$ by CMS.

the energy gap between fixed-target and previous HERA results, the exclusive cross section rises slowly with energy as $W^{0.13}$, consistent with Regge-phenomenology predictions. Interestingly, the proton-dissociative cross section is constant or decreases with $W$, which could be interpreted as a sign of saturation of the proton structure in a hot-spot model (see a plenary talk [50]), after the data are corrected for possible kinematic biases, e.g. due to the $M_{Y}<10 \mathrm{GeV}$ cut.

A. Bruni showed [51] the new ZEUS measurement of the ratio of exclusive $\psi(2 S)$ to $J / \psi$ cross sections in photoproduction [52], using the full HERA-II dataset. The new measurement, shown in red in Fig. 5 (middle), complements the measurement in DIS [53], and is the most-precise-to-date result at $Q^{2}=0$, exhibiting a potential to further constrain parameters of theoretical models.

The new measurement of (forward) exclusive photoproduction of the $J / \psi$ and $\psi(2 S)$ mesons in $p p$ collision at $13 \mathrm{TeV}$ by LHCb [54] were covered in the talk of M. Kucharczyk [55]. The analysis extends the earlier LHCb measurement at $7 \mathrm{TeV}$ [56], and benefits from using the HeRSCheL shower counters, installed upstream and downstream of the LHCb detector, which significantly reduce backgrounds. The cross sections are measured up to $W=3 \mathrm{TeV}$, in the high-W region the $J / \psi$ measurement (Fig. 6 right) is lower than a power-law extrapolation of H1 data. Both results favour NLO over LO predictions of the JMRT model [57, 58] (see Fig. 6 left for the $\psi(2 S)$ ).

A. Bylinkin presented [59] CMS measurements of exclusive photoproduction of the $\rho^{0}$ [60] and $\Upsilon$ [61] mesons in $p \mathrm{~Pb}$ UPCs at $5 \mathrm{TeV}$, in which lead ions serve as a rich source of photons. The exclusive $\rho^{0}$ signal is selected by requiring no signal in the Zero Degree Calorimeters, the cross section is measured as a function of $W$ and $|t|$ for $30<W<210 \mathrm{GeV}$ and $|t|<1 \mathrm{GeV}^{2}$. The result agrees with HERA data (Fig. 6 middle), and extends the HERA measurements to higher values of $|t|$. The $\Upsilon$ cross section is measured in the region $90<W<830 \mathrm{GeV}$ and fills the gap between the HERA and LHCb measurements (Fig. 6 right). A power-law fit to the data with $W^{\delta}$ yields $\delta=0.76 \pm 0.14$. The data has potential to constrain theory predictions.

Two talks reported studies of coherent $J / \psi$ photoproduction in photon-nucleus UPCs, sensitive to nuclear gluon distributions. J. Adam presented [62] the STAR measurement in Au+Au collisions at $200 \mathrm{GeV}$ at RHIC, with $J / \psi \rightarrow e e$ produced at central rapidities and UPC events selected by requiring neutrons from electromagnetic excitation of gold ions in Zero Degree Calorimeters. An interesting feature of the measurement is that, although not yet fully unfolded, the differential cross section vs. $|t|$ (Fig. 7 left) shows a sign of diffractive dip around $|t|=0.02 \mathrm{GeV}^{2}$, whose position is reproduced by dipole-based theory models. E. Kryshen [63] showed the new ALICE result on coherent $J / \psi$ production at forward rapidities in $\mathrm{Pb}+\mathrm{Pb}$ UPC at $5 \mathrm{TeV}$ [64]. The analysis is based on statistics that is factor of 200 higher than the previous ALICE result at $2.76 \mathrm{TeV}$ [65], and benefits from gap tagging in the ADA and ADC very forward detectors. The results are compared 

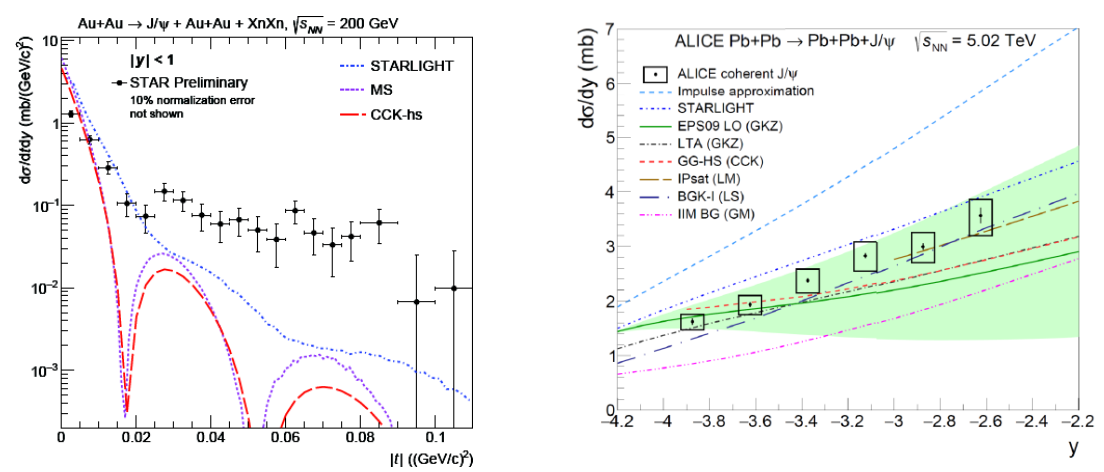

Figure 7: Coherent $J / \psi$ photoproduction measured (left) as a function of $|t|$ by STAR in Au+Au UPCs at $200 \mathrm{GeV}$, and (right) as a function of the $J / \psi$ rapidity measured by ALICE in Pb+Pb UPCs at $7 \mathrm{TeV}$.

to various theory predictions (Fig. 7 right), which suggest moderate nuclear gluon shadowing.

Finally, four talks covered rare exclusive processes induced by photon-photon fusion. The CMS result on diphoton production in the so-called light-by-light scattering process $(\gamma \gamma \rightarrow \gamma \gamma)$ was reported by A. Bylinkin [59]. The mesurement is based on the $\mathrm{Pb}+\mathrm{Pb}$ UPC data at $5 \mathrm{TeV}$ [66], in which 14 signal events are selected with a back-to-back diphoton topology $\left(A_{\phi}=1-\Delta \phi_{\gamma \gamma} / \pi<\right.$ 0.01 in Fig. 8 left) with $4.1 \sigma$ significance. The result is in good agreement with the first ATLAS measurement of $\gamma \gamma \rightarrow \gamma \gamma$ based on a similar dataset at $5 \mathrm{TeV}$ [67]. The newly updated ATLAS analysis [69] based on a factor-of-four higher statistics was presented by S. Hassani [68]. In the signal region $\left(A_{\phi}<0.005\right) 42$ events are observed with significance $8.2 \sigma$. The cross section for photons with $p_{T}>3 \mathrm{GeV}$, diphoton mass $m_{\gamma \gamma}>6 \mathrm{GeV}$ and $p_{T}^{\gamma \gamma}<1 \mathrm{GeV}$ is measured to be $\sigma_{\gamma \gamma \rightarrow \gamma \gamma}=78 \pm 13$ (stat) \pm 8 (syst) nb, compared to predicted $45-51 \mathrm{nb}$ (Fig. 8 middle). ATLAS also reported high-mass dimuon production in pp collisions at $13 \mathrm{TeV}$ [70]. The cross section of $\sigma_{\gamma \gamma \rightarrow \mu \mu}=3.12 \pm 0.07$ (stat) \pm 0.14 (syst) nb is measured for $m_{\mu \mu}>10 \mathrm{GeV}$, in agreement with QED predictions corrected for proton absorptive effects (of about 20\%). A. Vilela Pereira showed [71] the first CMS-TOTEM measurement of high mass dilepton production $\left(m_{l l}>110 \mathrm{GeV}, l=\mu, e\right)$ in $p p$ collisions at $13 \mathrm{TeV}$ [72], with the scattered proton measured in the CMS-TOTEM Precision Proton Spectrometer (CT-PPS) operating in standard high-luminosity conditions at the LHC. In the sample corresponding to an integrated luminosity of $9 \mathrm{fb}^{-1}, 12$ dimuon and 8 dielectron events are observed with significance $5.1 \sigma$ (Fig. 8 right). M. Tasevsky presented [73] a feasibility study of searches for dark matter in $p p$ collisions at $13 \mathrm{TeV}$, with the proton measured in the ATLAS Forward Proton detector, and assuming an integrated luminosity of $300 \mathrm{fb}^{-1}$ [74].
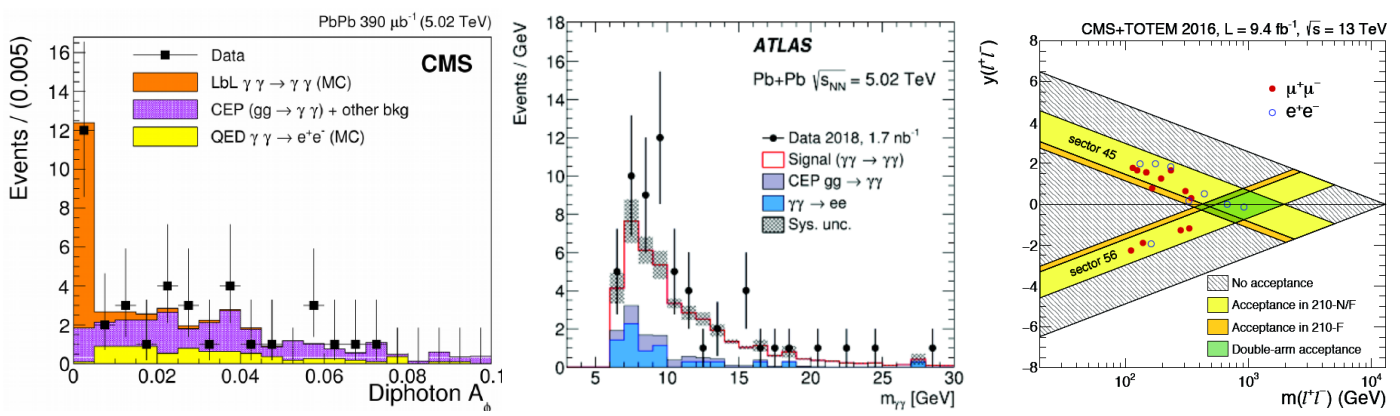

Figure 8: Light-by-light scattering events measured by (left) $\mathrm{CMS}$ and (middle) ATLAS in $\mathrm{Pb}+\mathrm{Pb}$ UPCs at $5 \mathrm{TeV}$. Right: Exclusive $\gamma \gamma \rightarrow l l$ events $(l=\mu, e)$ measured by CT-PPS in pp collisions at $13 \mathrm{TeV}$. 


\section{References}

[1] https://indico.cern.ch/event/749003/timetable/\#all.detailed.

[2] A. Watanabe, Differential and total cross sections of high energy proton-proton scattering in holographic QCD, these proceedings, PoS(DIS2019)078 [arXiv:1907.01416 [hep-ph]].

[3] D. Britzger, C. Ewerz, S. Glazov, O. Nachtmann and S. Schmitt, arXiv:1901.08524 [hep-ph].

[4] B. Ducloué, E. Iancu, A. H. Mueller, G. Soyez and D. N. Triantafyllopoulos, JHEP 1904 (2019) 081 [arXiv:1902.06637 [hep-ph]].

[5] J. Cepila, J. G. Contreras and M. Matas, Collinearly improved impact-parameter dependent Balitsky-Kovchegov evolution, these proceedings, PoS(DIS2019)255; Phys. Rev. D 99 (2019) no.5, 051502 [arXiv:1812.02548 [hep-ph]].

[6] B. I. Ermolaev and S. I. Troyan, Contribution of Double-Logarithmic Pomeron to structure function $F_{1}$, these proceedings, PoS(DIS2019)051; Eur. Phys. J. C 78 (2018) no.3, 204.

[7] G. A. Chirilli, Sub-eikonal spin corrections and $g_{1}$ structure function at low-x, these proceedings, PoS(DIS2019)253; JHEP 1901 (2019) 118 [arXiv:1807.11435 [hep-ph]].

[8] A. Dafne Bolognino, F. G. Celiberto, M. Fucilla, D. Y. Ivanov, B. Murdaca and A. Papa, Inclusive production of two rapidity-separated heavy quarks as a probe of BFKL dynamics, these proceedings, PoS(DIS2019)067 [arXiv:1906.05940 [hep-ph]].

[9] A. D. Bolognino, F. G. Celiberto, D. Y. Ivanov, M. M. A. Mohammed and A. Papa, High-energy effects in forward inclusive dijet and hadron-jet production, these proceedings, PoS(DIS2019)049 [arXiv:1906.11800 [hep-ph]].

[10] K. Golec-Biernat, L. Motyka and T. Stebel, Drell-Yan plus jet production and BFKL evolution, these proceedings, PoS(DIS2019)054; JHEP 1812 (2018) 091 [arXiv:1811.04361 [hep-ph]].

[11] T. Lappi and A. Ramnath, Unequal rapidity correlators in the dilute limit of JIMWLK, these proceedings, PoS(DIS2019)068; arXiv:1904.00782 [hep-ph].

[12] Z. Tu, D. E. Kharzeev and T. Ullrich, arXiv:1904.11974 [hep-ph].

[13] T. Altinoluk, P. Agostini and N. Armesto, Non-eikonal corrections to multi-particle production in the $C G C$, these proceedings, PoS(DIS2019)043; Eur. Phys. J. C 79 (2019) no.7, 600 [arXiv:1902.04483 [hep-ph]].

[14] E. Iancu and Y. Mulian, Nucl. Phys. A 985 (2019) 66 [arXiv:1809.05526 [hep-ph]].

[15] R. Maciula and A. Szczurek, Production of J/ $\psi$ quarkonia in color evaporation model based on $k_{T}$-factorization, these proceedings, PoS(DIS2019)066 [arXiv:1908.07429 [hep-ph]].

[16] M. Diehl and J. R. Gaunt, Adv. Ser. Direct. High Energy Phys. 29 (2018) 7 [arXiv:1710.04408 [hep-ph]].

[17] P. Kotko, L. Motyka, M. Sadzikowski and A. M. Stasto, arXiv:1905.00130 [hep-ph].

[18] A. H. Mueller and S. Munier, Phys. Rev. Lett. 121 (2018) no.8, 082001 [arXiv:1805.09417 [hep-ph]].

[19] C. A. Flett, S. P. Jones, A. D. Martin, M. G. Ryskin and T. Teubner, Towards a determination of the low $x$ gluon via exclusive J/ $\psi$ production, these proceedings, PoS(DIS2019)053 [arXiv:1907.06471 [hep-ph]]. 
[20] V. Skokov, Measuring the Weizsäcker-Williams distribution of linearly polarized gluons at an electron-ion collider through dijet azimuthal asymmetries, these proceedings, PoS(DIS2019)256 [arXiv:1907.10334 [hep-ph]].

[21] H. Mäntysaari, N. Mueller and B. Schenke, Diffractive dijet production from the Color Glass Condensate and the small-x gluon distributions, these proceedings, PoS(DIS2019)060 [arXiv:1906.04389 [hep-ph]].

[22] S. Bondarenko, On correlators of Reggeon fields in high energy $Q C D$, these proceedings, PoS(DIS2019)046; S. Bondarenko and S. Pozdnyakov, arXiv:1903.11288 [hep-th].

[23] M. Nefedov, One-loop corrections to multiscale effective vertices in the EFT for Multi-Regge processes in QCD, these proceedings, PoS(DIS2019)064 [arXiv:1905.01105 [hep-ph]].

[24] R. Zlebcik, Diffractive PDF determination from HERA inclusive and jet data at NNLO QCD, these proceedings, PoS(DIS2019)059

[25] D. Britzger, J. Currie, T. Gehrmann, A. Huss, J. Niehues and R. leb ík, Eur. Phys. J. C 78, 538 (2018) [arXiv:1804.05663 [hep-ph]].

[26] A. Aktas et al. [H1 Collaboration], JHEP 0710, 042 (2007) [arXiv:0708.3217 [hep-ex]].

[27] A. Stasto, N. Armesto, P.R. Newman and W. Slominski, Determination of diffractive parton densities at the LHeC and the FCC-eh, these proceedings, PoS(DIS2019)257

[28] N. Armesto, P. R. Newman, W. Slominski and A. M. Stasto, arXiv:1901.09076 [hep-ph].

[29] S. Chekanov et al. [ZEUS Collaboration], Nucl. Phys. B 831, 1 (2010) [arXiv:0911.4119 [hep-ex]].

[30] F.J. Nemes, Elastic and Total Cross-Section Measurements by TOTEM, these proceedings, PoS(DIS2019)065

[31] G. Antchev et al. [TOTEM Collaboration], CERN-EP-2017-335v3, arXiv:1812.04732 [hep-ex].

[32] G. Antchev et al. [TOTEM Collaboration], Eur. Phys. J. C 79, 103 (2019), arXiv:1712.06153 [hep-ex].

[33] G. Antchev et al. [TOTEM Collaboration], CERN-EP-2018-338, arXiv:1812.08283 [hep-ex].

[34] G. Antchev et al. [TOTEM Collaboration], CERN-EP-2018-341 arXiv:1812.08610 [hep-ex].

[35] V. M. Abazov et al. [D0 Collaboration], Phys. Rev. D 86, 012009 (2012) [arXiv:1206.0687 [hep-ex]].

[36] J. R. Cudell et al. [COMPETE Collaboration], Phys. Rev. Lett. 89, 201801 (2002) doi:10.1103/PhysRevLett.89.201801 [hep-ph/0206172].

[37] A. Szczurek and P. Lebiedowicz, Searching for odderon in exclusive reactions: $p p \rightarrow p p p \bar{p}$, $p p \rightarrow p p \phi \phi$ and $p p \rightarrow p p \phi$, these proceedings, PoS(DIS2019)071

[38] P. Lebiedowicz, O. Nachtmann and A. Szczurek, Phys. Rev. D 97, no. 9, 094027 (2018) [arXiv:1801.03902 [hep-ph]].

[39] P. Lebiedowicz, O. Nachtmann and A. Szczurek, Phys. Rev. D 99, no. 9, 094034 (2019) [arXiv:1901.11490 [hep-ph]].

[40] M. Tasevsky, Measurements of single diffraction using the ALFA forward spectrometer at ATLAS, these proceedings, PoS(DIS2019)072

[41] The ATLAS collaboration, ATLAS-CONF-2019-012.

[42] V. Khachatryan et al. [CMS Collaboration], Phys. Rev. D 92, no. 1, 012003 (2015) [arXiv:1503.08689 [hep-ex]]. 
[43] K. Kuznetsova, Recent CMS and CMS-TOTEM results on diffraction, these proceedings, PoS(DIS2019)058

[44] CMS Collaboration, CMS-PAS-FSQ-12-033.

[45] A. Ekstedt, R. Enberg and G. Ingelman, arXiv:1703.10919 [hep-ph].

[46] A. M. Sirunyan et al. [CMS Collaboration], Eur. Phys. J. C 78, 242 (2018) [arXiv:1710.02586 [hep-ex]].

[47] C. Dilks, Transverse Spin Asymmetries in the $p^{\uparrow} p \rightarrow p \pi^{0} X$ Process at STAR, these proceedings, PoS(DIS2019)050

[48] A. Bolz, Exclusive $\rho(770)$ photoproduction at HERA, these proceedings, PoS(DIS2019)045

[49] H1 Collaboration, H1prelim-18-012.

[50] J.G. Contreras, Some recent results related to low-x and forward physics, these proceedings, PoS(DIS2019)274

[51] A. Bruni, Measurement of the Psi(2S) to J/Psi cross section ratio in photoproduction with the ZEUS detector at HERA, these proceedings, PoS(DIS2019)047

[52] ZEUS Collaboration, ZEUS-prel-18-003 (https://inspirehep.net/record/1680705).

[53] H. Abramowicz et al. [ZEUS Collaboration], Nucl. Phys. B 909, 934 (2016) [arXiv:1606.08652 [hep-ex].

[54] R. Aaij et al. [LHCb Collaboration], JHEP 1810, 167 (2018) [arXiv:1806.04079 [hep-ex]].

[55] M. Kucharczyk, Soft QCD and Central Exclusive Production at LHCb, these proceedings, PoS(DIS2019)057

[56] R. Aaij et al. [LHCb Collaboration], J. Phys. G 41, 055002 (2014) [arXiv:1401.3288 [hep-ex]].

[57] S. P. Jones, A. D. Martin, M. G. Ryskin and T. Teubner, JHEP 1311, 085 (2013) [arXiv:1307.7099 [hep-ph]].

[58] S. P. Jones, A. D. Martin, M. G. Ryskin and T. Teubner, J. Phys. G 41, 055009 (2014) [arXiv:1312.6795 [hep-ph]].

[59] A. Bylinkin, Recent CMS results on exclusive processes, these proceedings, PoS(DIS2019)048

[60] A. M. Sirunyan et al. [CMS Collaboration], Eur. Phys. J. C 79, 702 (2019), arXiv:1902.01339 [hep-ex].

[61] A. M. Sirunyan et al. [CMS Collaboration], Eur. Phys. J. C 79, 277 (2019) [arXiv:1809.11080 [hep-ex]].

[62] J. Adam, Coherent J/ $\psi$ photoproduction in ultra-peripheral collisions at STAR, these proceedings, PoS(DIS2019)042

[63] E. Kryshen, Recent ALICE results on coherent J/psi photoproduction in ultra-peripheral Pb-Pb collisions, these proceedings, PoS(DIS2019)056

[64] S. Acharya et al. [ALICE Collaboration], arXiv:1904.06272 [nucl-ex].

[65] B. Abelev et al. [ALICE Collaboration], Phys. Lett. B 718, 1273 (2013) [arXiv:1209.3715 [nucl-ex]].

[66] A. M. Sirunyan et al. [CMS Collaboration], Phys. Lett. B 797, 134826 (2019), arXiv:1810.04602 [hep-ex]. 
[67] M. Aaboud et al. [ATLAS Collaboration], Nature Phys. 13, no. 9, 852 (2017) [arXiv:1702.01625 [hep-ex]].

[68] S. Hassani, Photon-photon fusion measurements at ATLAS, these proceedings, PoS(DIS2019)055

[69] G. Aad et al. [ATLAS Collaboration], Phys. Rev. Lett. 123, no. 5, 052001 (2019) [arXiv:1904.03536 [hep-ex]].

[70] M. Aaboud et al. [ATLAS Collaboration], Phys. Lett. B 777, 303 (2018) [arXiv:1708.04053 [hep-ex]].

[71] A. Vilela Pereira, Results and prospects with the CMS-TOTEM Precision Proton Spectrometer, these proceedings, PoS(DIS2019)077

[72] A. M. Sirunyan et al. [CMS and TOTEM Collaborations], JHEP 1807, 153 (2018) [arXiv:1803.04496 [hep-ex]].

[73] M. Tasevsky, Searches for Dark Matter at the LHC in forward proton mode, these proceedings, PoS(DIS2019)073

[74] L. A. Harland-Lang, V. A. Khoze, M. G. Ryskin and M. Tasevsky, JHEP 1904, 010 (2019) [arXiv:1812.04886 [hep-ph]]. 